\title{
Numerical studies of the behavior of ionized residual gas in an energy recovering linac
}

\author{
Gisela Pöplau* and Ursula van Rienen \\ University of Rostock, Rostock, Germany
}

\author{
Atoosa Meseck ${ }^{\dagger}$ \\ HZB, Berlin, Germany
}

(Received 10 October 2014; published 29 April 2015)

\begin{abstract}
Next generation light sources such as energy recovering linacs (ERLs) are highly sensitive to instabilities due to ionized residual gas, which must be mitigated for successful operation. Vacuum pumps are insufficient for removal of the ions, as the ions are trapped by the beam's electrical potential. Two effective measures are (i) introducing clearing gaps in the bunch train, and (ii) installing clearing electrodes which pull out the trapped ions from the electrical potential of the beam. In this paper, we present numerical studies on the behavior of ion clouds that interact with bunch trains in an ERL taking into account the effects of the clearing gaps and clearing electrodes. We present simulations with different compositions of the residual gas. Simulations are done using the MOEVE PIC Tracking software package developed at Rostock University, which has been upgraded to include the behavior of ion clouds in the environment of additional electromagnetic fields, such as generated by clearing electrodes. The simulations use the parameters of the Berlin Energy Recovery Linac Project (bERLinPro) to allow for the deduction of appropriate measures for bERLinPro 's design and operation.
\end{abstract}

DOI: 10.1103/PhysRevSTAB.18.044401

PACS numbers: 41.20.Cv, 41.75.Lx, 29.27.Bd

\section{INTRODUCTION}

In an electron accelerator, several effects such as collision with the electron beam, synchrotron radiation and field emission can lead to ionization of the rest gas in the beam pipe. The positive ions can be trapped in the negative electrical potential of the electron beam and lead in turn to an increase of the beam halo, to emittance blowup and to transverse and longitudinal instabilities by interacting (oscillating) resonantly with the beam. There are several measures for avoiding the ion-trapping such as utilizing clearing electrodes or using short or long clearing gaps.

Clearing electrodes seem to be simple and goal-oriented as they pull out the trapped ions from the vicinity of the beam. However, as they superimpose additional electric fields to the electromagnetic field configuration given by the magnets and the transitory bunches, detailed investigations on their optimum shapes and maximum voltages in connection with the expected gas mixtures are mandatory.

\footnotetext{
*Present address: Compaec e. G., Rostock, Germany. gisela.poeplau@uni-rostock.de

Also at Physics Department, Humboldt University, Berlin, Germany.

atoosa.meseck@helmholtz-berlin.de

Published by the American Physical Society under the terms of the Creative Commons Attribution 3.0 License. Further distribution of this work must maintain attribution to the author(s) and the published article's title, journal citation, and DOI.
}

A long clearing gap between two trains of highrepetition rate bunches allows for ions to drift out of the beam potential and thus to reach the wall of the vacuum chamber, while a short clearing gap causes large oscillations of ions around the beam which in turn clear the vicinity of the bunches from the ions. Please note that clearing gaps in an energy recovering linac (ERL) do not mitigate the fast ion instability, as they do not preclude ions from accumulating in a single bunch train and interacting resonantly with the electrons in that train $[1,2]$. However, the applicability of the long-gap approach in ERLs suffers from the transient effects of the rf systems [1]. The short clearing gaps on the other hand seem to be favorable, in particular as experimental and numerical studies indicate that multi-bunch trains with short gaps are very effective in suppressing the ion trapping process [3]. Furthermore, in an ERL the transient rf effects can be avoided by ensuring that the clearing gaps in the accelerating and in the decelerating beam coincide. Although short clearing gaps in the ERLbeam allow for suppression of the ion accumulation in the vicinity of the beam, they are not totally welcome because they change the continuous wave $(\mathrm{CW})$ characteristics of the radiation delivered by the ERL. Therefore, to keep the time characteristic as $\mathrm{CW}$-like as possible, frequent but extremely short bunch gaps are favorable (CW means here, a beam consisting of equidistant pulses coming with a repetition rate up to $\mathrm{GHz}$ ).

In the presented work, we introduce a newly implemented numerical method for the study of the behavior of 
ionized residual gas, where the fields of clearing electrodes as well as the electrical potential of transitory electron bunches are included. It allows for the 3D tracking of different particle species including the computation of the full 3D space charge fields. Thus, we are able to estimate clearing times, to undertake detailed studies of the behavior of the ions under the influence of the fields of the electrodes with different voltage and the transitory bunch, and to investigate the impact of the different long clearing gaps. The starting point for this new implementation was the software package MOEVE PIC Tracking developed in Rostock originally for the simulation of single bunch instabilities caused by an electron cloud [4-6]. The software package has been further developed to model the interaction of the ions with the electron beam in presence of external electromagnetic potentials such as the field of clearing electrodes $[7,8]$.

For the numerical simulations presented, we have used the parameters planned for bERLinPro described in [9] as an example. The parameters relevant for the simulations in this paper are summarized in Table I. With the exception of the beam energy, these parameters are very close to those of the Cornell X-ray ERL where in an analytical study it has been shown that ions are a critical issue for the optimum operation of the ERL [1]. Please note that the beam energy has an indirect influence on the beam ion interaction via transverse beam size. Similar to the Cornell ERL, for machine settings envisaged for bERLinPro, all ions have an atomic mass larger than the critical mass (please, see next section for the definition) and are therefore trapped in the electrical potential of the bunch. Thus, following [1] one can expect that the degradation of the beam quality caused by the ions is a serious threat to an optimum operation of bERLinPro, making detailed analytical and numerical investigations essential for the design of the facility. In this paper, we especially consider the influence of different compositions of the residual gas on the clearing time. We also study the impact of clearing gaps with different lengths. In particular we investigate whether a bunch gap as short as one accelerating bucket occurring very frequently can excite oscillations large enough to clear the vicinity of the beam.

The paper is organized as follows: a brief introduction of the analytical formulas describing the physics of beam-ion-instability and ion-clearing process in the

TABLE I. Nominal parameters of bERLinPro.

\begin{tabular}{lc}
\hline \hline Nominal Beam Energy & $50 \mathrm{MeV}$ \\
Average Beam Current $I$ & $100 \mathrm{~mA}$ \\
Nominal Beam Charge $Q$ & $77 \mathrm{pC}$ \\
Maximum Repetition Rate & $1.3 \mathrm{GHz}$ \\
Normalized Emittance & $10^{-6} \mathrm{~m}$ \\
Bunch Length $\sigma_{t}$ & $2 \mathrm{ps}$ \\
Vacuum Pressure & $10^{-10} \mathrm{mbar}$ \\
\hline \hline
\end{tabular}

second section of the paper is followed by a detailed description of the simulation tool MOEVE PIC Tracking in Sec. III. The numerical results are detailed in Sec. IV followed by the discussion and concluding remarks in Sec. V.

\section{BEAM-ION-INSTABILITY AND ION-CLEARING PROCESS}

As discussed above, several effects can lead to ionization of the rest gas in the accelerator environment. The negative potential of the electron beam captures then the positive ions, leading either to scattering processes which increase the beam halo and blow up the emittance or to transverse and longitudinal coupled ion-beam oscillations which also can blow up the emittance and lead to beam losses. Therefore, clearing out the pipe of ions is mandatory. In a high repetition rate ERL, additional measures such as clearing electrodes and clearing gaps are needed in order to sufficiently clear out the pipe of the ions. In this section, analytical formulas describing the process of ion accumulation, the ion oscillation and the clearing process are provided.

\section{A. Ionization accumulation and ion oscillation}

The positively charged ions are attracted to the electron beam. Ions with an atomic mass larger than the critical mass [see Eq. (1)] will be trapped in the electrical potential of the beam. They start to oscillate around the electron beam, thereby exciting oscillations of the electron bunches in turn. For the oscillation frequency of the ion cloud there are several theoretical results available $[3,10]$.

Let $\lambda_{e}$ denote the line charge of the bunch, $r_{p}$ the classical proton radius, $c$ the speed of light in vacuum, $N_{b}$ the number of electrons per bunch, $L_{\text {sep }}$ the bunch separation, $A$ the relative atomic mass of the ion, $\sigma_{i, x}, \sigma_{i, y}$ the transversal dimension of the ion cloud and $\sigma_{e, x}, \sigma_{e, y}$ the transversal rms bunch size.

The critical mass, $A_{\text {crit }}$, is defined by

$$
A_{\text {crit }}=\frac{N_{b} r_{p} L_{\mathrm{sep}}}{\sigma_{e, x}\left(\sigma_{e, x}+\sigma_{e, y}\right)} .
$$

Due to the large beam size and the small charge of the bunches envisaged for bERLinPro $A_{\text {crit }}$ is less than one, thus all the ions will be captured in the electron beam potential.

The frequency of the ion oscillation $\omega_{i, x}$ according to Chao [10] is

$$
\omega_{i, x, y}=c\left(\frac{2 \lambda_{e} r_{p}}{A \sigma_{e, x, y}^{2}}\right)^{1 / 2}
$$

where it is assumed that the ions are produced at rest and that they have a uniform distribution. 
Wang et al. [3] give the expression

$$
\omega_{i, x, y}=c\left[\frac{2 \lambda_{e} r_{p}}{A k_{x, y} \sigma_{i, x, y}\left(\sigma_{i, x}+\sigma_{i, y}\right)}\right]^{1 / 2},
$$

where the parameter $k_{x, y}$ is given by

$$
k_{x, y}=\frac{\Sigma_{x, y}\left(\Sigma_{x}+\Sigma_{y}\right)}{\sigma_{e, x, y}\left(\sigma_{e, x}+\sigma_{e, y}\right)}
$$

with $\Sigma_{x, y}=\sqrt{\sigma_{e, x, y}^{2}+\sigma_{i, x, y}^{2}}$. Assuming $\sigma_{e, x, y}^{2}=\sigma_{i, x, y}^{2} / 2$ the parameter $k_{x, y}$ yields $k_{x, y}=3 / 2$. Although these results are obtained with a Gaussian distribution of the ions in the cloud the authors of [3] point out that the ion cloud's transversal distribution has a peak-like shape. This is confirmed by our simulations using a parameter set similar to that of bERLinPro (see Fig. 1).

Please note that the ions in the cloud repel each other. So far in the literature, these repulsive forces have been neglected in the analytical considerations, as they complicate the calculations further. However, in the presented studies they are included showing interesting aspects of the motions of ions in the vicinity of the electrodes and the bunch.

\section{B. Clearing electrodes}

The electric force generated by the static fields of these electrodes pulls the ions out of the beam's potential. For this purpose, the voltage of the electrodes has to be sufficiently large. For the computation of the potential $U$ in the center of the bunch we consider the formula given in [11]:

$$
U=\frac{I}{2 \pi \varepsilon_{0} \beta c}\left[\ln \left(\frac{r_{c}}{a}\right)-\frac{1}{2}\right] .
$$

Here, $I$ denotes the current, $\varepsilon_{0}$ the vacuum permittivity, $\beta$ the ratio of the velocity and the velocity of light $c, r_{c}$ the radius of the vacuum chamber and $a$ the rms radius of the beam, respectively. Note that the potential $U$ denotes a potential at the transverse center of the beam and thus virtually at the transverse center of the beam pipe. Being positioned on the chamber wall, the electrodes have a distance of $r_{c}$ to the beam center, so that their voltage needs to be a factor $\left(r_{c} / a\right)^{2}$ higher to equal the beam potential. For the extraction of trapped ions from the beam, the voltage of the electrodes needs to be even higher than the beam potential. For bERLinPro clearing electrodes, depending on the location a voltage of about $800 \mathrm{~V}$ to $1000 \mathrm{~V}$ is necessary to equal the beam potential. Increasing these voltages by a factor three, we assume that a reasonably fast ion extraction is enabled. Averaging over all possible electrode locations, an average clearing voltage of $U \approx-2625 \mathrm{~V}$ is calculated for $a=0.7 \mathrm{~mm}$ and $r_{c}=2 \mathrm{~cm}$.
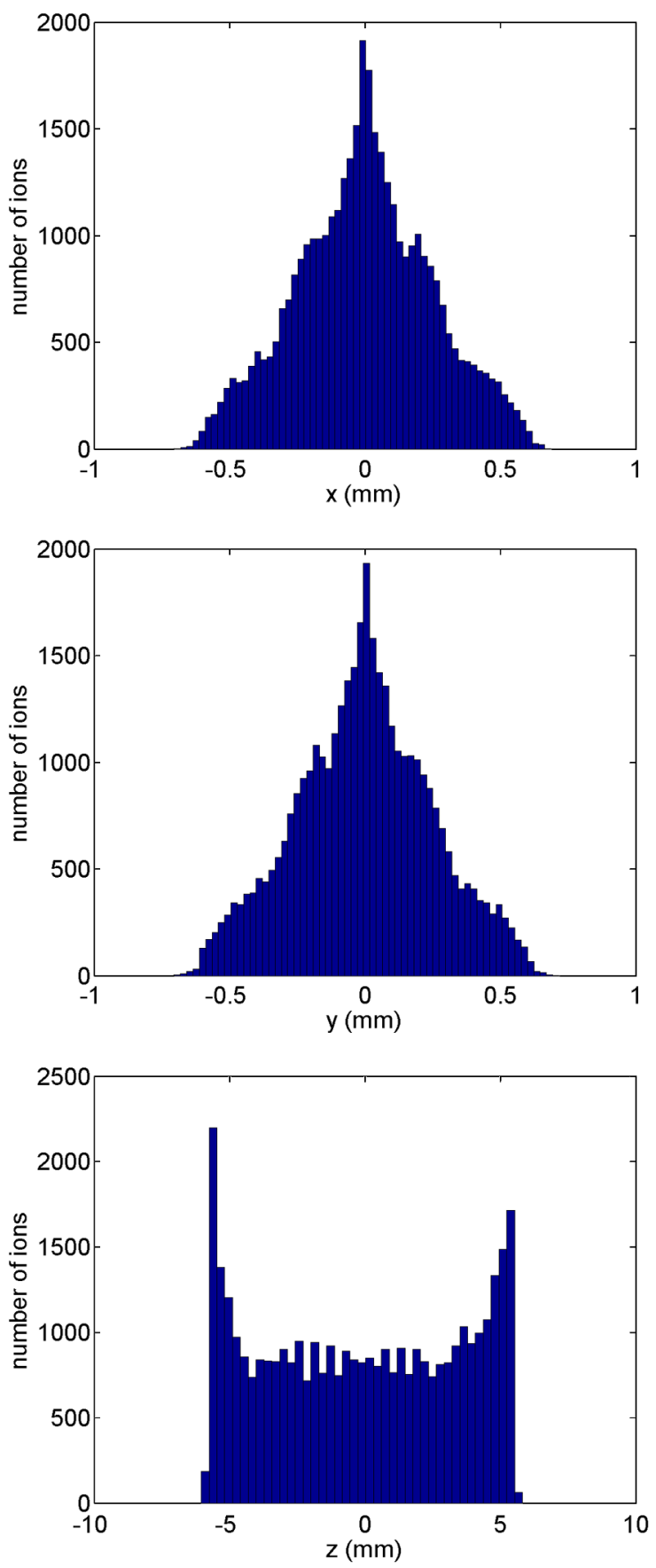

FIG. 1. Distribution of $\mathrm{H}_{2}^{+}$ions after 10,000 interactions with a $100 \mathrm{MeV}$ bunch.

\section{Clearing gaps}

Assuming that the ions are generated with near thermal velocities, it is a simple conclusion that they are not free to drift in the vacuum chamber. They experience at the one hand the space charge potential of the electron beam as long as the beam is not fully neutralized and at the other hand the repulsive forces of the neighboring ions. Of course there are also additional external magnetic fields such as dipole and quadrupole fields which we omit in the presented study. 
Their effects on the ions are subject to ongoing studies [12]. The beam potential attracts the ions toward the center of the beam thus causing the previously discussed transverse oscillations of the ions with an amplitude given by their transverse position at "birth" [13].

A gap in the bunch train is a significant change in the field configuration of the entire system of beam and ions. In contrast to a long clearing gap which allows for the ions to fully escape the beam potential and reach the chamber wall, in a short clearing gap the ions have not enough time to escape the beam. However, the short gap gives the beam potential a time structure. This leads to a time dependent attractive force with many frequency components which in turn excites large ion oscillations clearing the vicinity of the beam $[1,3]$.

However, also very short clearing gaps change the CW characteristics of the electron beam. In order to keep the time characteristic as $\mathrm{CW}$-like as possible, frequent but extremely short bunch gaps are favorable. This raises the question whether a bunch gap as short as one bunch occurring very frequently can excite oscillations large enough to clear the vicinity of the beam.

\section{SIMULATION TOOL MOEVE PIC TRACKING}

The simulation tool MOEVE PIC Tracking allows for the computation of the interaction of the ion cloud with the bunch and includes the field generated by the clearing electrodes [8]. It has been developed at the University of Rostock for the tracking of charged particles taking into account the 3D space charge fields in each tracking step $[6,7]$.

Hence, this approach allows for very detailed numerical studies of the dynamics of ion clouds beyond established analytical results $[1,11,14]$.

For the determination of the self-induced space charge fields the particle-mesh method [15] is implemented in MOEVE PIC Tracking. Hereby, a computational domain $\Omega$ is constructed around the particles. In our model $\Omega$ is transversally determined by the wall of the vacuum chamber and longitudinally by some additional space around the particle cloud. For this domain Poisson's equation has to be solved to obtain the potential $\varphi$ by

$$
\begin{aligned}
-\Delta \varphi & =\frac{\varrho}{\varepsilon_{0}} \text { in } \Omega \subset \mathbb{R}^{3}, \\
\varphi & =0 \quad \text { on } \partial \Omega_{1}, \\
\varphi & =\varphi_{0} \quad \text { on } \partial \Omega_{2},
\end{aligned}
$$

where $\varrho$ denotes the charge distribution and $\varepsilon_{0}$ the permittivity in vacuum. In the presented simulations, the domain $\Omega$ of our model is a short piece of a beam pipe with elliptical cross-section. On the surface of the pipe a perfectly conducting boundary is assumed $(\varphi=0)$. Additionally, $\varphi=0$ is chosen in longitudinal direction in order to achieve a stable solution algorithm. This assumption is satisfied if the boundary is located far enough from the particle cloud. All boundary parts with $\varphi=0$ are summarized by $\partial \Omega_{1}$. Boundary $\partial \Omega_{2}$ denotes the location of the electrodes with a given potential $\varphi_{0}$.

The numerical solution of Eq. (6) is performed with the following steps: (i) discretization of Eq. (6) by second order finite differences, (ii) distribution of the particle's charge to the mesh points, (iii) numerical solution of the resulting system of equations by a multigrid preconditioned conjugate gradient method [7], (iv) determination of the electric field at the grid points by

$$
\mathbf{E}=-\nabla \varphi,
$$

(v) interpolation of the field values that are available at the grid points to the position of the particles. Considering the interaction, the field values of ions have to be interpolated to the position of both macro electrons and ions, whereas the field generated by the bunch has to be interpolated to the position of the ions [compare Eqs. (11) and (12) below].

As the number of electrons per bunch is usually more than $10^{8}$, the concept of macro-particles is applied for the electrons, summarizing a large number of electrons in a macro-electron. The steps described above are applied separately for each particle species: the macroelectrons of the bunch and the ions. It is worth mentioning that the Poisson equation for the bunch has to be solved in the rest frame of the bunch and that the resulting field has to be transformed back to the laboratory frame. Furthermore, the field of the electrodes is not considered for the bunch, i.e., the boundary condition simplifies to

$$
\varphi=0 \quad \text { on } \partial \Omega .
$$

The interaction between a bunch and the ion cloud is computed within the tracking step as follows. Let $\mathbf{E}_{\mathrm{b}}$ and $\mathbf{E}_{\mathrm{i}}$ denote the electric field generated by the bunch and by the ions, further $\mathbf{v}_{\mathrm{b}}$ and $\mathbf{v}_{\mathrm{i}}$ the velocity of the bunch's particles and the velocity of the ions, respectively. Then, the force acting on the bunch's particles is given by

$$
\mathbf{F}_{\mathrm{b}}=q\left(\mathbf{E}_{\mathrm{b}}+\mathbf{E}_{\mathrm{i}}+\mathbf{v}_{\mathrm{b}} \times \frac{\mathbf{v}_{\mathrm{b}} \times \mathbf{E}_{\mathrm{b}}}{c^{2}}\right)
$$

and the force acting on the ions is given by

$$
\mathbf{F}_{\mathrm{i}}=q\left(\mathbf{E}_{\mathrm{b}}+\mathbf{E}_{\mathrm{i}}+\mathbf{v}_{\mathrm{i}} \times \frac{\mathbf{v}_{\mathrm{b}} \times \mathbf{E}_{\mathrm{b}}}{c^{2}}\right) .
$$

Now assuming that the bunch moves longitudinally nearly with the speed of light $\mathbf{v}_{\mathrm{b}} \approx c$, then $\mathbf{v}_{\mathrm{b}} \times \frac{\mathbf{v}_{\mathrm{b}} \times \mathbf{E}_{\mathrm{b}}}{c^{2}}=$ $-\mathbf{E}_{\mathrm{b}}$ and the force on the bunch simplifies to 


$$
\mathbf{F}_{\mathrm{b}}=q \mathbf{E}_{\mathrm{i}}
$$

Further, considering Eq. (10) we can assume $\mathbf{v}_{\mathrm{i}} \ll \mathbf{v}_{\mathrm{b}}$ and obtain for the force on the ions

$$
\mathbf{F}_{\mathrm{i}}=q\left(\mathbf{E}_{\mathrm{b}}+\mathbf{E}_{\mathrm{i}}\right) .
$$

After the interaction the ions are tracked further taking into account only the field induced by the ions until the next bunch arrives and the interaction is computed again.

\section{NUMERICAL RESULTS}

For the evaluation and a deeper understanding of the numerical results presented in this work, the details of modeling the electron and ion distributions as well as the exact description of the individual simulations are essential. Therefore, the following section starts with the details of particle modeling and the benchmarking test verifying the applicability of the upgraded code, and then presents the results of the numerical studies.

\section{A. Modeling the initial bunch and ion cloud}

The models for bunch and ion cloud are based on 3D particle distributions. The particle distribution of the bunch is generated by means of the procedure generator of ASTRA [16] as a distribution of 100,000 macro-electrons with Gaussian distribution in each direction representing a total charge of $77 \mathrm{pC}$. Energy and emittance are chosen due to Table I.

The ions are distributed uniformly with respect to position. Each component of the velocity vector $\left(v_{i}, i=1,2,3\right)$ is determined by the Maxwell-Boltzmann distribution given by

$$
f\left(v_{i}\right)=\sqrt{\frac{m}{2 \pi k_{B} T}} \cdot \mathrm{e}^{-\frac{m v_{i}^{2}}{2 k_{B} T}}, \quad i=1,2,3 .
$$

Here, $m$ denotes the mass of the ion species, $k_{B}=1.38 \times 10^{-23} \mathrm{~J} \mathrm{~K}^{-1}$ the Boltzmann constant and $T$ the temperature taken with $T=300 \mathrm{~K}$.

For the studies ion clouds with different ion species are utilized. The data of these ion cloud compositions are summarized in Table II. For the first test case the ion cloud consists only of $\mathrm{H}_{2}^{+}$. The second composition (mixture 2) corresponds to studies for the Cornell X-ray ERL [1]. Mixture 3 was taken due to measurements at SPEAR3 [17]. The main difference of these last two compositions lies in the percentage of the ions much heavier than $\mathrm{H}_{2}^{+}$: for mixture 2 it is $2 \%$ and in mixture 3 it is 52\%. Mixture 4-6 are taken from recent simulations of the possible profiles of the vacuum pressure in bERLinPro under three different conditions [18]. Here the vacuum pressures vary from $4.0 \times 10^{-10}$ to $3.5 \times 10^{-12}$ mbar due to different chamber coatings and
TABLE II. Mixtures of ionized residual gas used for the simulations.

\begin{tabular}{lccc}
\hline \hline & ion species & percentage & mass number \\
\hline mixture 1 & $\mathrm{H}_{2}^{+}$ & $100 \%$ & 2 \\
mixture 2 & $\mathrm{H}_{2}^{+}$ & $98 \%$ & 2 \\
& $\mathrm{CH}_{4}^{+}$ & $1 \%$ & 16 \\
& $\mathrm{CO}^{+}$ & $1 \%$ & 28 \\
mixture 3 & $\mathrm{H}_{2}^{+}$ & $48 \%$ & 2 \\
& $\mathrm{CH}_{4}^{+}$ & $5 \%$ & 16 \\
& $\mathrm{H}_{2} \mathrm{O}^{+}$ & $16 \%$ & 18 \\
& $\mathrm{CO}^{+}$ & $14 \%$ & 28 \\
& $\mathrm{CO}_{2}^{+}$ & $17 \%$ & 44 \\
mixture 4 & $\mathrm{H}_{2}^{+}$ & $5.0 \%$ & 2 \\
& $\mathrm{CH}_{4}^{+}$ & $1.3 \%$ & 16 \\
& $\mathrm{CO}^{+}$ & $93.7 \%$ & 28 \\
mixture 5 & $\mathrm{H}_{2}^{+}$ & $28.0 \%$ & 2 \\
& $\mathrm{CH}_{4}^{+}$ & $0.4 \%$ & 16 \\
& $\mathrm{CO}^{+}$ & $71.6 \%$ & 28 \\
mixture 6 & $\mathrm{H}_{2}^{+}$ & $48.0 \%$ & 2 \\
& $\mathrm{CH}_{4}^{+}$ & $26.0 \%$ & 16 \\
& $\mathrm{CO}^{+}$ & $26.0 \%$ & 28 \\
\hline \hline
\end{tabular}

pump configuration. In comparison to the first three mixtures, the percentage of heavy $\mathrm{CO}^{+}$ions is much higher in the last three mixtures. Table III summarizes the gas pressures and corresponding number of ions in the clouds for the mixtures detailed in Table II.

\section{B. Benchmarking test: Ion oscillation}

A first benchmarking of the upgraded software tool MOEVE PIC Tracking with analytical formulas is performed by the numerical calculations of the oscillation of an ion cloud around the electron beam, confirming the formulas of Eq. (2) and (3). The simulations are performed with electron bunches of uniform and Gaussian distribution with an energy of $50 \mathrm{MeV}$ and $100 \mathrm{MeV}$, respectively. The ion cloud is modeled as a cloud of $\mathrm{H}_{2}^{+}$ions distributed longitudinally in a $1 \mathrm{~cm}$ long section with a transversal extension of $\sigma_{i, x, y}^{2}=2 \sigma_{e, x, y}$. The ions have uniform distribution in all space directions while their velocities are Boltzmann distributed as described in subsection IVA. Assuming a vacuum pressure of $1 \times 10^{-10} \mathrm{mbar}$ the ion cloud is modeled with 85,000 ions for the $50 \mathrm{MeV}$ bunch

TABLE III. Pressures of residual gas and resulting number of ions per $\mathrm{cm}$.

\begin{tabular}{lccc}
\hline \hline & $\begin{array}{c}\text { pressure } \\
{[\mathrm{mbar}]}\end{array}$ & $\begin{array}{c}\sharp \text { ions } \\
{[\mathrm{M}]}\end{array}$ & $\begin{array}{c}\# \text { simulated } \\
\text { ions [M] }\end{array}$ \\
\hline mixture 1-3 & $1.000 \times 10^{-10}$ & 30.36 & 1.0 \\
mixture 4 & $4.000 \times 10^{-10}$ & 212.5 & 2.125 \\
mixture 5 & $0.280 \times 10^{-10}$ & 14.88 & 1.488 \\
mixture 6 & $0.035 \times 10^{-10}$ & 1.86 & 1.86 \\
\hline \hline
\end{tabular}




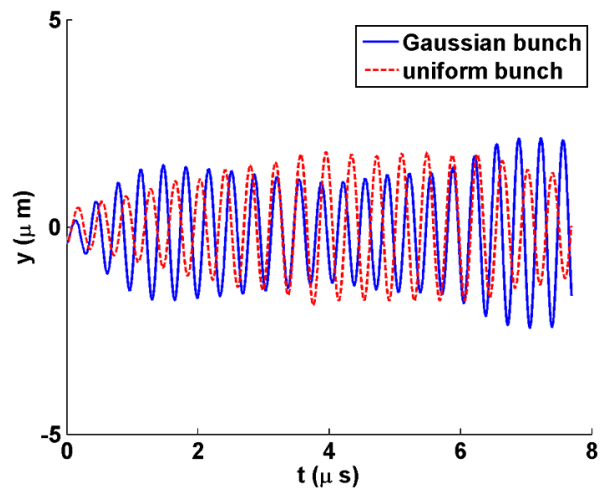

FIG. 2. Vertical oscillation of an ion cloud consisting of $\mathrm{H}_{2}^{+}$ ions around the beam potential of a $50 \mathrm{MeV}$ bunch.

and with 38,000 ions for the $100 \mathrm{MeV}$ bunch, respectively. The difference arises from the fact that assuming the same normalized emittance as envisaged for bERLinPro the transverse beam size is different for different energies.

Figures 2-5 give examples of the simulation results. The oscillations of the vertical position of a $\mathrm{H}_{2}^{+}$ion cloud around the beam potential of a $50 \mathrm{MeV}$ and a $100 \mathrm{MeV}$ beam are shown in Figs. 2 and 4, respectively. The corresponding Fourier transforms are represented in Figs. 3 and 5. The results of the benchmarking test are summarized in Table IV, where we have taken the frequency at the maximum amplitude. The simulation and theory agree very well, as a comparison of the simulation result of the Gaussian bunch with Eq. (3) of Wang and the uniform bunch with Eq. (2) of Chao shows, although the simulated frequency is in all cases smaller than the theoretical value.

From the very good agreement between our simulation and the analytical formulas, we conclude that the upgraded software correctly models the beam-ion-interaction.

\section{Numerical studies with clearing electrodes}

As described in the last section, the ion clouds are modeled with the mixtures specified in Table II. The ions

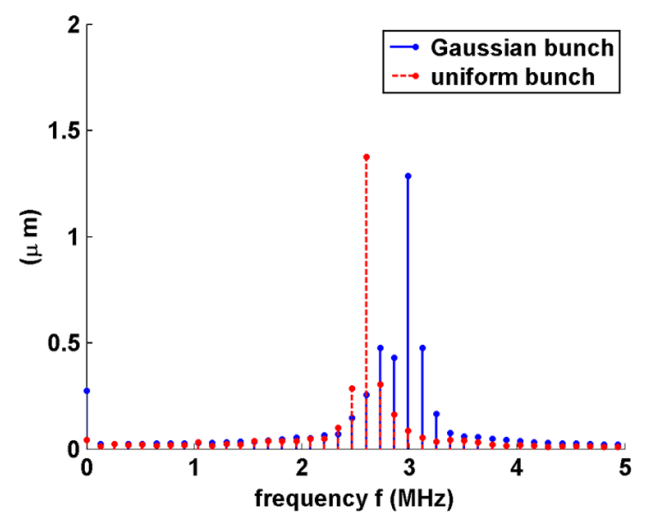

FIG. 3. Fourier analysis of the oscillation of a $\mathrm{H}_{2}^{+}$ion cloud around the beam potential of a $50 \mathrm{MeV}$ bunch.

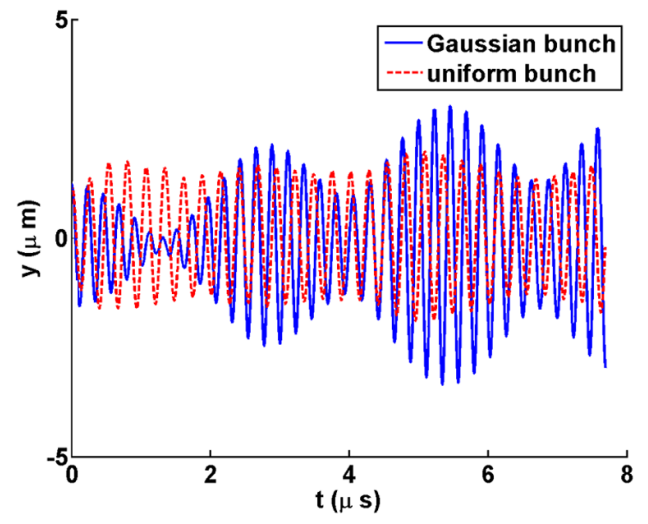

FIG. 4. Vertical oscillation of an ion cloud consisting of $\mathrm{H}_{2}^{+}$ ions around the beam potential of a $100 \mathrm{MeV}$ bunch.

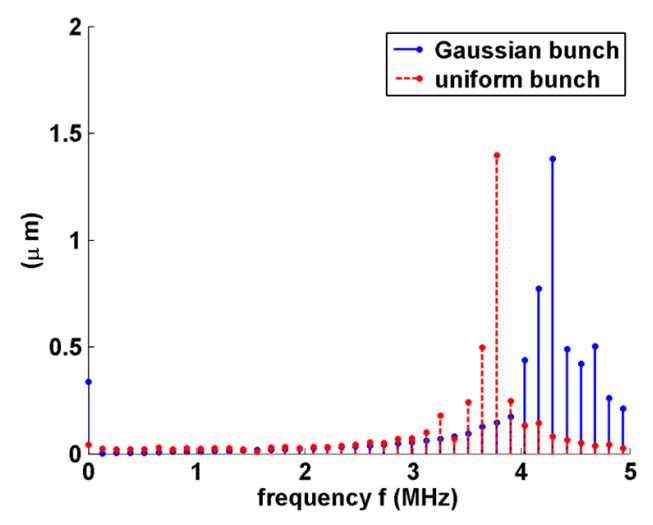

FIG. 5. Fourier analysis of the oscillation of a $\mathrm{H}_{2}^{+}$ion cloud around the beam potential of a $100 \mathrm{MeV}$ bunch.

are distributed over the whole pipe's cross-section along a length of $1 \mathrm{~cm}$ and $2 \mathrm{~cm}$, respectively. The beam pipe is modeled either as a circular pipe with a radius of $2 \mathrm{~cm}$ or as an elliptical pipe with a semi-major axis of $3.5 \mathrm{~cm}$ and a semi-minor axis of $2 \mathrm{~cm}$. The electrodes are button-like with a diameter of $16 \mathrm{~mm}$. The electrodes are located on opposite sides of the beam pipe and the voltage of each is set to the same value of $-2700 \mathrm{~V}$ due to the observations in [8].

TABLE IV. Frequency of ion oscillation: theoretical and numerical results compared for $\mathrm{H}_{2}^{+}$ions. Repetition rate of the bunch is $1.3 \mathrm{GHz}$.

\begin{tabular}{lcccc}
\hline \hline & & \multicolumn{2}{c}{ Simulation } \\
\cline { 3 - 5 } & \multicolumn{1}{c}{ Wang } & Chao & Gauss & uniform \\
\hline $50 \mathrm{MeV}$ & & & \\
$f$ & $3.15 \mathrm{MHz}$ & $2.72 \mathrm{MHz}$ & $2.99 \mathrm{MHz}$ & $2.6 \mathrm{MHz}$ \\
$t$ & $0.32 \mu \mathrm{s}$ & $0.37 \mu \mathrm{s}$ & $0.33 \mu \mathrm{s}$ & $0.38 \mu \mathrm{s}$ \\
$100 \mathrm{MeV}$ & & & \\
$f$ & $4.41 \mathrm{MHz}$ & $3.82 \mathrm{MHz}$ & $4.29 \mathrm{MHz}$ & $3.77 \mathrm{MHz}$ \\
$t$ & $0.23 \mu \mathrm{s}$ & $0.26 \mu \mathrm{s}$ & $0.23 \mu \mathrm{s}$ & $0.28 \mu \mathrm{s}$ \\
\hline \hline
\end{tabular}




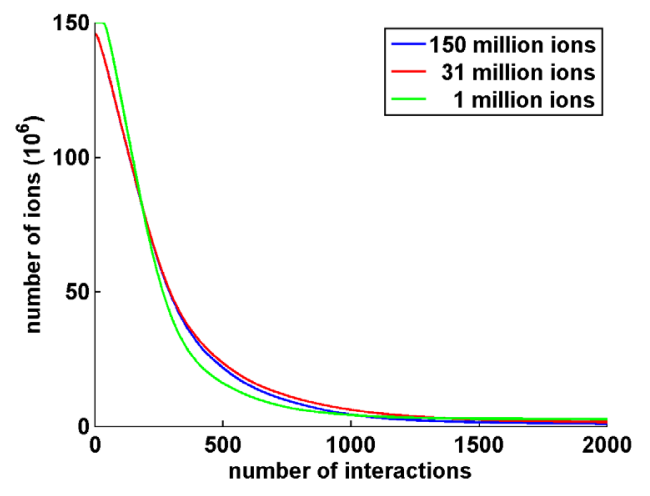

FIG. 6. Clearing times of ion clouds with $\mathrm{H}_{2}^{+}$ions cleared by the field of electrodes with a voltage of $-2700 \mathrm{~V}$. Please note that in the cases of $31 \mathrm{M}$ and $1 \mathrm{M}$ ions the vertical axis is scaled up for the sake of better visibility.

With regard to the CPU-time and the computational resources, it is important to determine the number of ions that need to be taken into account for the clearing electrodes simulations. Assuming that all molecules of the residual gas are ionized a pipe with a radius of $2 \mathrm{~cm}$ is filled with 31 million ions per $\mathrm{cm}$ at a vacuum pressure of $10^{-10}$ mbar. Simulation results for these parameters have been presented in [8]. From experiences in accelerator operation this pressure can be related to a maximum pressure of $10^{-8}$ mbar in worst case, which corresponds to 3,100 million ions per $\mathrm{cm}$ for the related simulations. That in turn is impossible to run on a normal PC. Hence, the question is what can be concluded from a simulation with, e.g., 1 million ions for the dynamics of 31 million ions and more.

As a test, we compare the results from simulations with ion clouds - consisting only of $\mathrm{H}_{2}^{+}$- of 1 million, 31 million (corresponds to a vacuum pressure of $10^{-10} \mathrm{mbar}$ ) and 150 million ions (corresponds to a vacuum pressure of ca. $5 \times 10^{-10} \mathrm{mbar}$ ), respectively. Figure 6 presents the

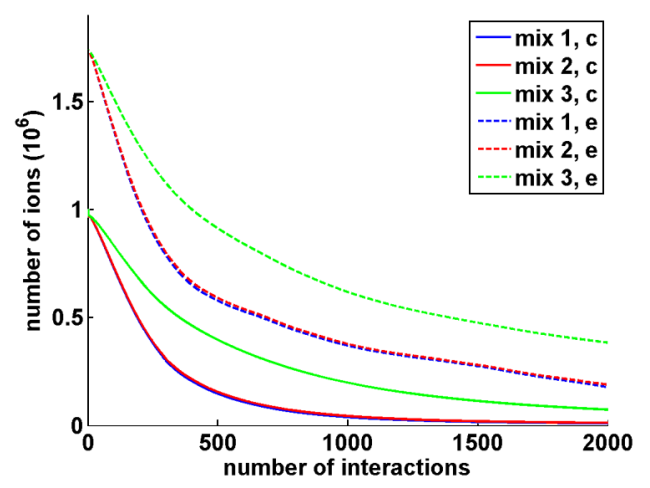

FIG. 7. Clearing times of ion clouds with different compositions in a circular (solid lines) and elliptical (dashed lines) beam pipe. 2000 bunch-interactions correspond to a clearing time of about $1.5 \mu \mathrm{s}$.

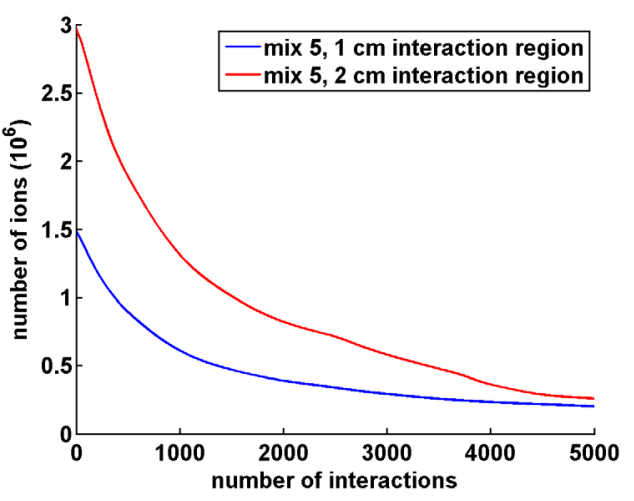

FIG. 8. Clearing times of ion clouds with different width: $1 \mathrm{~cm}$ and $2 \mathrm{~cm}$ of mixture 5 , beam pipe with elliptical cross-section.

clearing time for these ion clouds scaled up to 150 million in order to make the results comparable. It turns out that independent of the number of ions most of the ions are cleared after $1 \mu \mathrm{s}$. As a consequence we perform the simulations of mixed ions only with a total number of 1 million ions. Figure 7 shows the clearing time for ionized residual gas with compositions according to Table II. As the elliptical beam pipe has larger dimensions, the corresponding simulations start with a higher number of ions. After a clearing time of about $3 \mu \mathrm{s}$ an equilibrium like state is reached. As shown exemplary in Fig. 8 for the mixture 5, independent of the extent of the simulation-window (here $1 \mathrm{~cm}$ and $2 \mathrm{~cm}$, respectively) about the same number of ions remains in the vicinity of the beam. It seems as if the clearing time increases with increasing percentage of heavier ions. Repeating the simulations for the mixtures $4-6$ in a circular beam pipe (see Fig. 9) verifies that as expected the clearing time increases with increasing percentage of heavier ions in the ionized residual gas.

Figure 10 shows the evolution of the ion cloud with mixture 2 within the first $\mu \mathrm{s}$, where only $\mathrm{H}_{2}^{+}$(dark blue) and

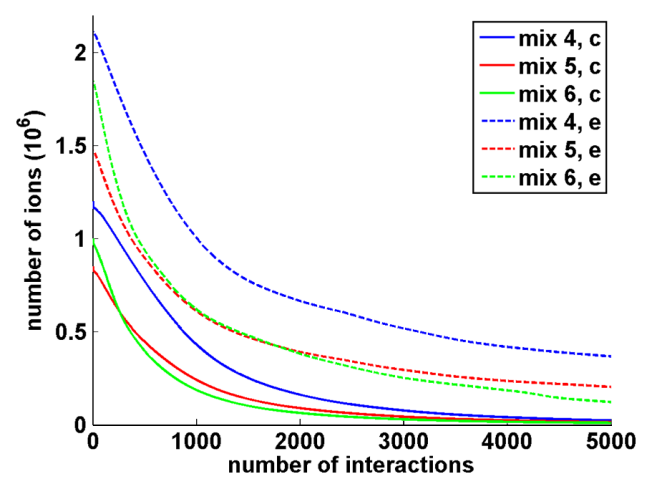

FIG. 9. Clearing times of ion clouds with different compositions (mixture 4-6) in a circular beam pipe. 5000 bunchinteractions correspond to a clearing time of about $4 \mu \mathrm{s}$. 


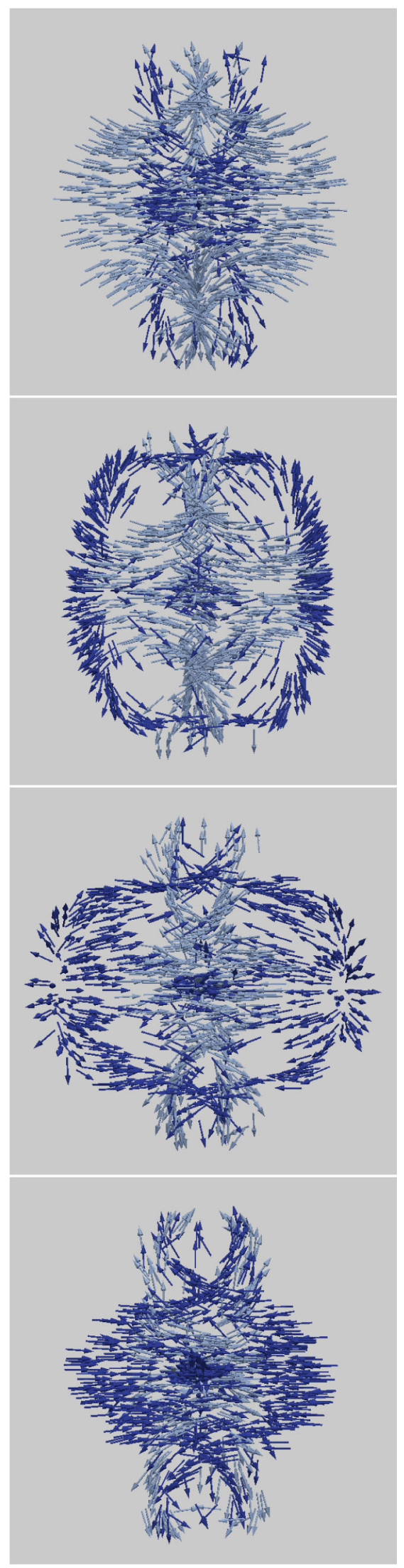

FIG. 10. Evolution of an ion cloud (dark blue: $\mathrm{H}_{2}^{+}$, light blue: $\mathrm{CH}_{4}^{+}$) in the field of electrodes with a voltage of $-2700 \mathrm{~V}$ after $0.23 \mu \mathrm{s}, 0.38 \mu \mathrm{s}, 0.54 \mu \mathrm{s}$ and $0.69 \mu \mathrm{s}$ (from top).
$\mathrm{CH}_{4}^{+}$(light blue) are plotted. The originally uniformly distributed ion species are separated due to their different mass and consequently different velocity. All ions are attracted by the field of the electrodes as well as by the field of the bunch. In the beginning mainly the ions in the neighborhood of the electrodes are cleared. Later in the clearing process more and more ions in the vicinity of the beam are attracted to the electrodes. The field of the electrodes snatches away the remaining ions from the beam. Moving fast (in comparison to the heavier ions of course) toward the electrodes, the $\mathrm{H}_{2}^{+}$ions (dark blue) are cleared first, while the $\mathrm{CH}_{4}^{+}$ions (light blue) seem rather frozen on their positions. Figure 11 shows the different
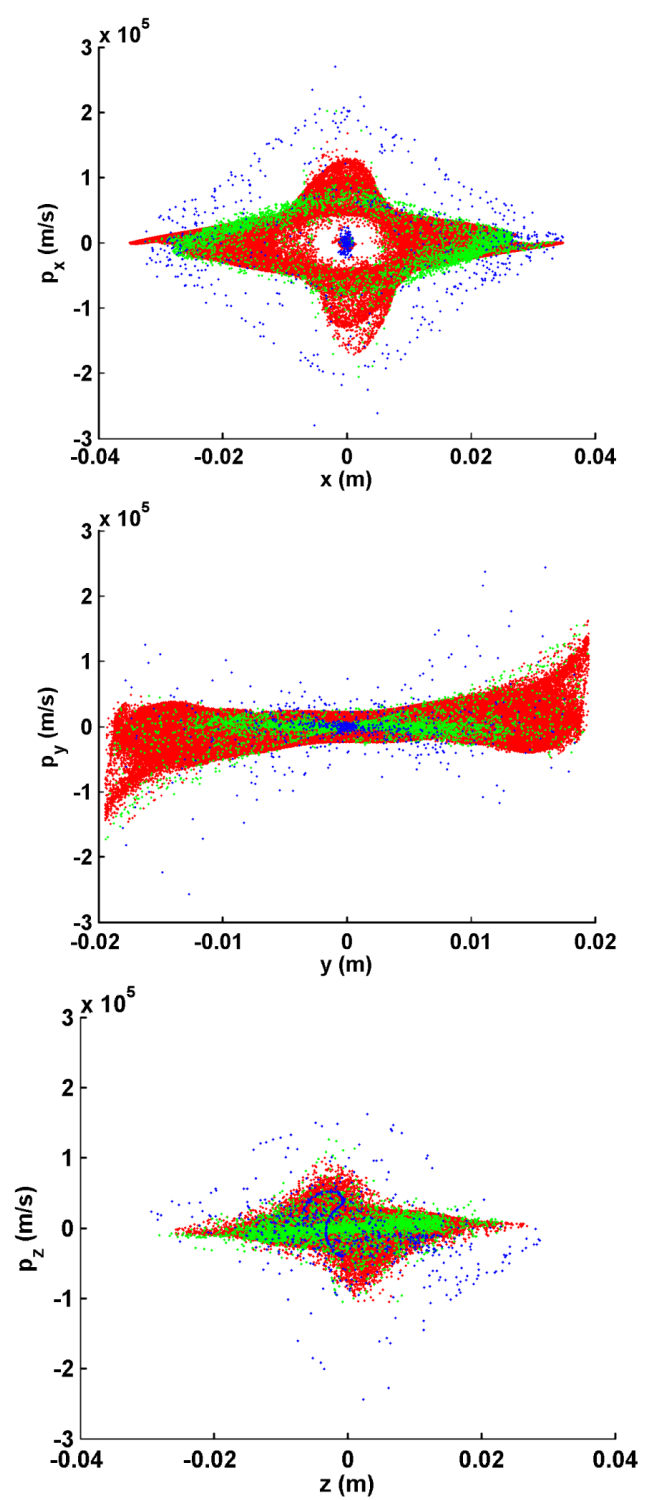

FIG. 11. Distribution of the ions in mixture 4 in phase space $\left(x, p_{x}\right)$ (top), $\left(y, p_{y}\right)$ (middle) and $\left(z, p_{z}\right)$ (bottom) after 5000 interactions with the electron bunch, $\mathrm{CO}^{+}$-red, $\mathrm{CH}_{4}^{+}$- green, $\mathrm{H}_{2}^{+}$- blue. 

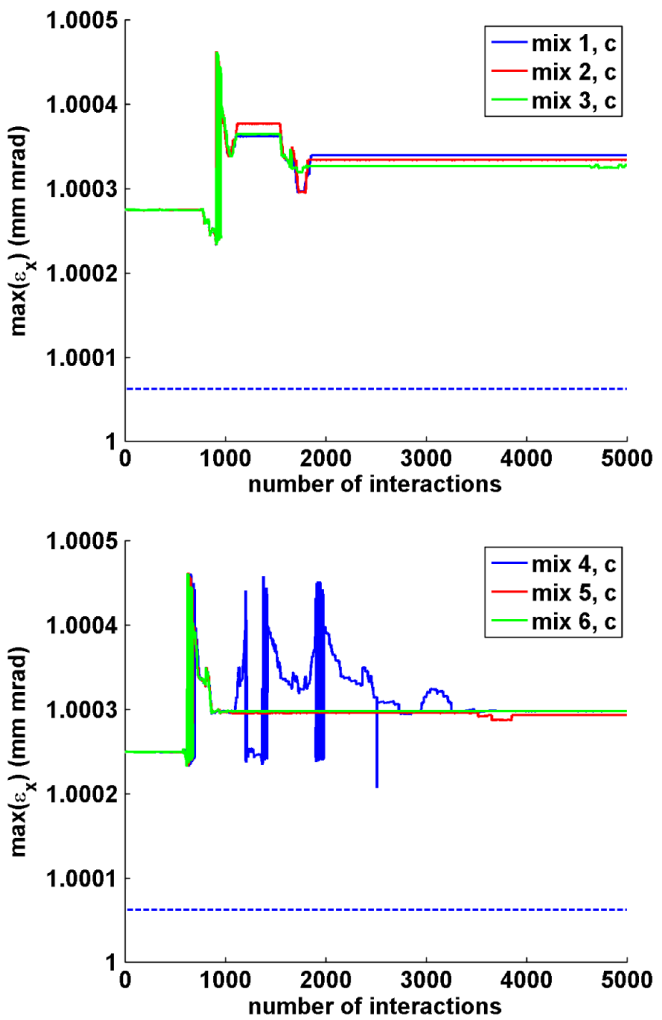

FIG. 12. Maximal emittance of a bunch after interaction with different composition of ion clouds in a circular beam pipe. The dotted line indicates the designed emittance.

phase-space distributions after 5000 interactions with the electron bunch for mixture 4. Please note that also in this case the initial ion distribution is uniform for all ions. The clearing electrodes are positioned vertically, as can also be concluded from the vertical phase-space distribution. The electron beam axis is at $(0,0)$. The ions obtain strong vertical momenta aimed toward the electrodes while their horizontal momenta aim in different directions. The overall motion of the ions is a spiraled motion

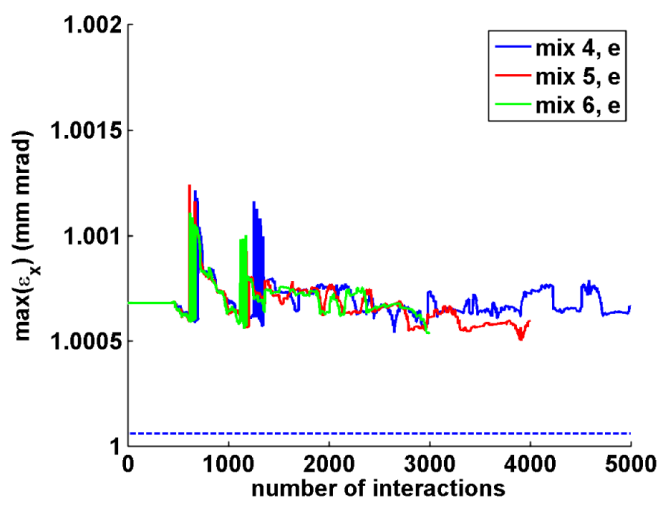

FIG. 13. Maximal emittance of a bunch after interaction with different composition of ion clouds (mixture 4-6) in an elliptical beam pipe. The dotted line indicates the designed emittance.

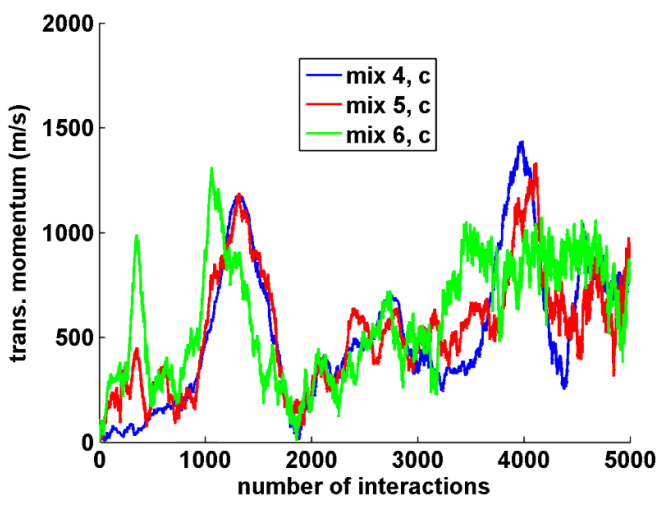

FIG. 14. Average transverse momentum of different ion clouds: mixture 4-6 in a circular beam pipe.

toward the electrodes. The lighter $\mathrm{H}_{2}^{+}$ions are almost fully removed, while the much heavier $\mathrm{CO}^{+}$ions remain in the pipe.

As explained previously, after some time the field of the electrodes snatches away the ions from the vicinity of the beam. The change in the slope of the curves in Fig. 7 marks the onset of this process. It coincides with the oscillations of the horizontal emittance $\varepsilon_{x}$ shown in Fig. 12. Since the direct impact of the clearing field on the electrons remains unchanged in these simulations, the change of the field configuration is only due to the significant reduction of ions in the vicinity of the beam. After some time-the extent of which depends on the specific ion mixture - the majority of the remaining ions are located inside the bunch area (see Fig. 10 bottom). The oscillations in the values of the emittance vanish at some level in the case of the circular beam pipe (Fig. 12) or get much smaller within the elliptical beam pipe (Fig. 13). Please note that due to the finite extensions of the clearing electrodes and the different diameters of the simulated beam pipes, the clearing forces in the elliptical case are smaller than in the circular case. Consequently, the momentum of ions is much larger in the

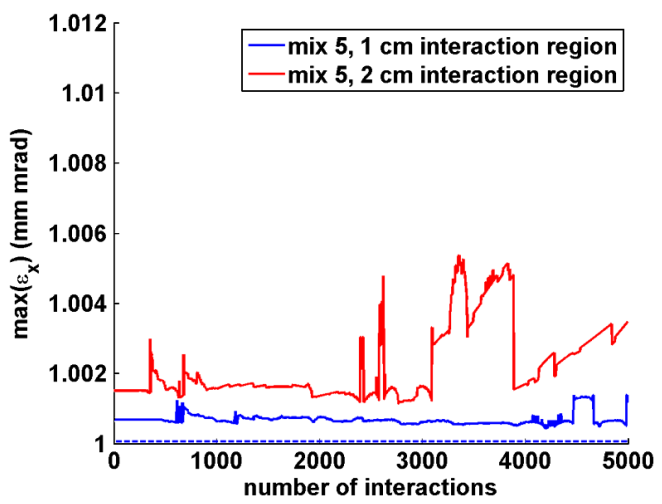

FIG. 15. Maximal emittance of a bunch after interaction with ion clouds of different width: $1 \mathrm{~cm}$ and $2 \mathrm{~cm}$ of mixture 5 . The dotted line indicates the designed emittance. 


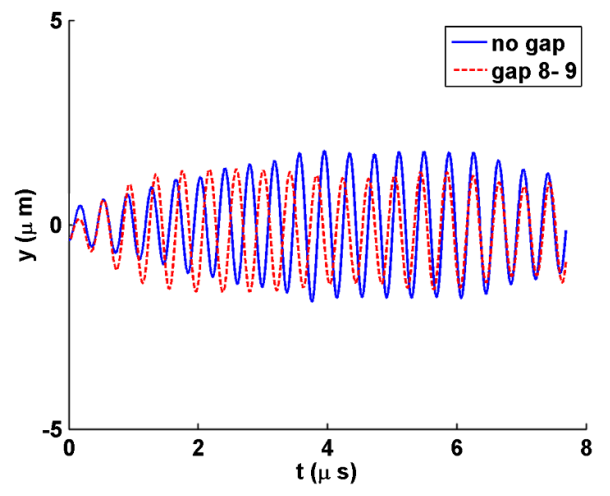

FIG. 16. Oscillation of an ion cloud ( $y$-component) interacting with a full bunch train and a bunch train with two gaps at each 8th and 9th position of the train.

circular case as shown in Fig. 14. Furthermore, in case of the elliptical beam pipe we stopped the computations after 4000 interactions with the electron bunch for mixture 5 and after 3000 interactions for mixture 6 , because the number of remaining $\mathrm{H}_{2}^{+}$ions is small, the average value has a large numerical noise so that our model is not valid anymore (see Fig. 13). The impact on the vertical emittance is quite small for all mixtures.

Extending the simulation-window as shown, for example, in Fig. 15 for mixture 5, has a visible impact on the emittance as it changes the dynamics of the intra ion interaction. However, the overall behavior remains in the sense that the features of the variation in emittance are similar despite differing scales depending on the size of the interaction region.

\section{Numerical studies with clearing gaps}

In this section we study the impact of the effect of clearing gaps of different length on an ion cloud. All ion clouds are modeled with $\mathrm{H}_{2}^{+}$as described in Sec. IV B. Further a $50 \mathrm{MeV}$ bunch with uniform distribution is

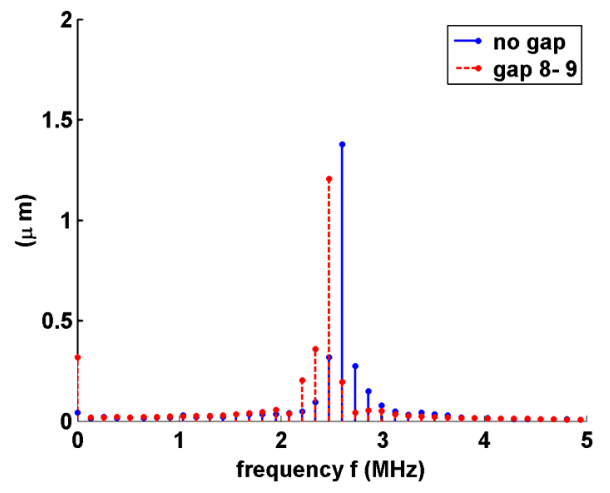

FIG. 17. Fourier analysis of the oscillation of an ion cloud interacting with a full bunch train and a bunch train with a 100 bunch gap from the 900th to 999th position in the train.

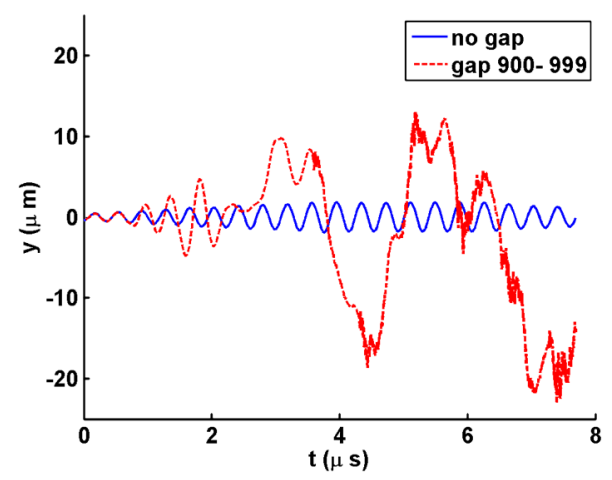

FIG. 18. Oscillation of an ion cloud (y-component) interacting with a full bunch train and a bunch train with 100 gaps between each 900th and 999th position of the train.

assumed. The impact of single-bucket clearing gaps in a bunch train have already been investigated in [19]. In those previous studies different longitudinal distributions for the particles in the bunch and different spacings of the clearing gap have been investigated. It was concluded that although the single-bucket-gap clearly leads to an increase of the ion oscillation amplitudes around the beam, the excited amplitudes are not large enough to clear the beam vicinity of ions.

The impact of a short, two bucket long clearing gap as well as the impact of a longer gap of 100 buckets is presented in this work. In the simulations, the short gap occurs at each 8th bucket, while the long gap appears at each 900th bucket. Figures 16 and 17 show comparisons of the vertical oscillations of the ion clouds with and without the gaps and the corresponding Fourier analysis, respectively. It can be observed that the oscillation frequency in the case of a two-bucket clearing gap is lower than the oscillation frequency of the cloud interacting with a completely filled bunch train, but the amplitude of the excited oscillation is still not sufficiently large for ions to escape the beam potential.

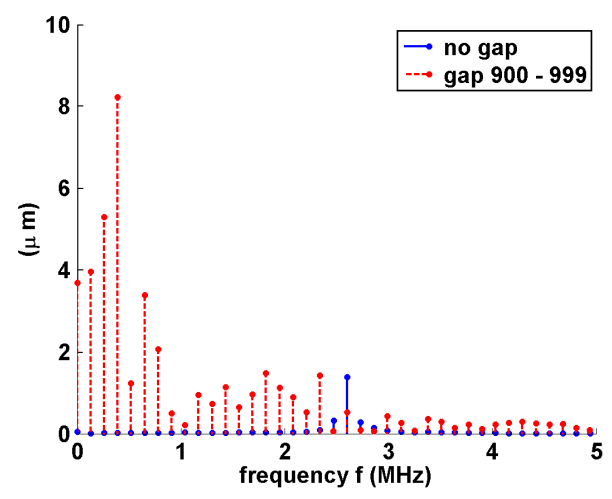

FIG. 19. Fourier analysis of the oscillation of an ion cloud interacting with a full bunch train and a bunch train with 100 gaps between each 900th and 999th position of the train. 


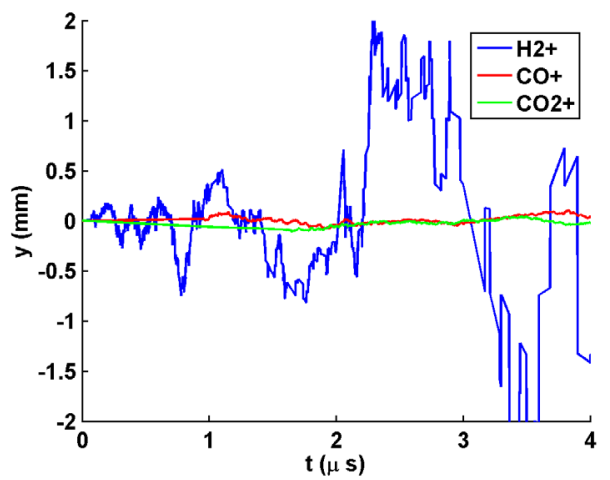

FIG. 20. Oscillation of ion clouds ( $y$-component) consisting of $\mathrm{H}_{2}^{+}, \mathrm{CO}^{+}, \mathrm{CO}_{2}^{+}$ions around the beam potential of a $50 \mathrm{MeV}$ bunch with Gaussian distribution and a repetition rate of $1 \mathrm{MHz}$.

The situation changes for a longer gap of 100 bunches. Figure 18 presents the vertical oscillations. In this case, the gap is large enough, so that the amplitude of the oscillations increases remarkably. Hence, the transversally accelerated ions can escape the bunch potential, reach the wall of the vacuum chamber, and get neutralized. The corresponding Fourier analysis plotted in Fig. 19 shows that in contrast to a completely filled bunch train in this case many lower frequencies are excited.

In a third case we study the impact of the repetition frequency on the bunch. For this we change the bunch charge to keep the average current fixed and set the repetition rate to $1 \mathrm{MHz}$. This changes the bunch separation in Eq. (1). Accordingly, all ions of the residual gas can escape from the attracting force of the beam potential. Clouds of three different ion species are investigated: $\mathrm{H}_{2}^{+}$, $\mathrm{CO}^{+}, \mathrm{CO}_{2}^{+}$. Figure 20 shows the oscillations of these ions in the vertical direction. As expected the $\mathrm{H}_{2}^{+}$ions move fast and reach the wall of the vacuum chamber. According to the simulations all $\mathrm{H}_{2}^{+}$ions are neutralized after 7.24 $\mu \mathrm{s}$. Since $\mathrm{CO}^{+}$and $\mathrm{CO}_{2}^{+}$ions are much heavier and hence slower it takes much longer to accelerate these ions. Thus, the clearing process starts later as shown in Fig. 21.

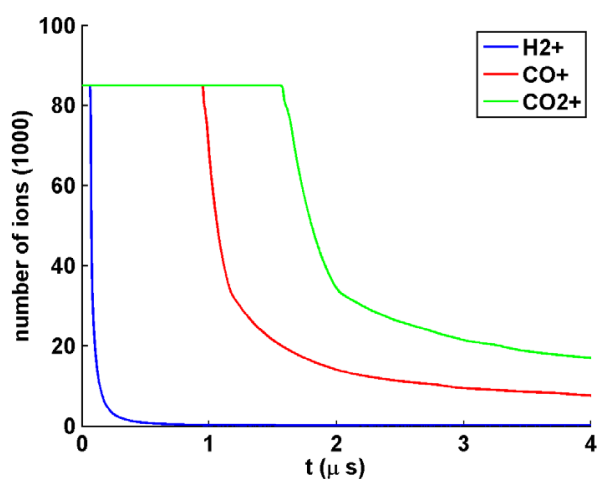

FIG. 21. Clearing times of ion clouds consisting of $\mathrm{H}_{2}^{+}, \mathrm{CO}^{+}$, $\mathrm{CO}_{2}^{+}$ions around the beam potential of a $50 \mathrm{MeV}$ bunch with Gaussian distribution and a repitition rate of $1 \mathrm{MHz}$.
Nevertheless all $\mathrm{CO}^{+}$ions are cleared after $36.3 \mu \mathrm{s}$ and the $\mathrm{CO}_{2}^{+}$ions after $50.5 \mu \mathrm{s}$.

\section{CONCLUSION}

In this paper, we present numerical studies on the behavior of ion clouds of different compositions interacting with bunch trains in an ERL. We take into account the effects of the clearing gaps and clearing electrodes. Furthermore, we provide detailed simulations of the behavior of the ions interacting with the electrical potential of the bunch and the field of the clearing electrodes simultaneously. For the numerical investigations, the software package MOEVE PIC Tracking developed at Rostock University has been used. The detailed numerical studies have been enabled by upgrading the code MOEVE PIC Tracking. Using analytical formulas for ion oscillation as benchmarking cases, we verify that the upgraded code models the bunch-ion interaction correctly. The presented numerical investigations take into account the parameters of the ERL bERLinPro.

From our simulation, we conclude that using clearing electrodes with a voltage as high as $-2700 \mathrm{~V}$, the vicinity of the beam can be sufficiently cleared. For a pressure on the order of $10^{-10}$ mbar, a clearing time of about a few $\mu \mathrm{s}$ leads to an equilibrium-like state. For this pressure the neutralization time in bERLinPro is about $10 \mathrm{~s}$ to $20 \mathrm{~s}$, thus significantly (7 orders of magnitude) bigger than clearing time. Decreasing the accelerator pressure by an order of magnitude to $10^{-11}$ mbar can allow for much lower clearing voltage while maintaining the same neutralization-time to clearing time ratio. Using NEC-coated vacuum chambers, which are available today, a pressure of $10^{-11}$ mbar and less seems within reach. However, independent of the extent of the simulation-window and the shape of the simulated pipe about the same number of ions remain in the vicinity of the beam. It seems as if the clearing time and the percentage of remaining ions increase with the increasing percentage of heavier ions in the residual gas composition. The impact on the beam emittance is more complex and can only be fully investigated after including the external magnetic fields in the code. This is the subject of ongoing studies.

While a short clearing gap of a few buckets excites only small changes in the oscillation amplitude of the ions clouds, a longer clearing gap in order of 100 buckets can clear out the ions very efficiently. A reduction of the bunch repetition rate to $1 \mathrm{MHz}$ prevents the ion trapping and is thus a very effective remedy. However a reduction of the repetition rate as well as a long clearing gap might not be very attractive in operation of an ERL, therefore the usage of clearing electrodes in the locations where the electrical potential of the beam is maximal-e.g., location with minimal transverse beam size - seems to be the best option to counteract the impact of the ions in bERLinPro. 


\section{ACKNOWLEDGMENTS}

This work was supported by the Federal Ministry of Education and Research (BMBF) under Contract No. 05K10HRC. The authors would like to thank A. Markovik (University of Rostock) for providing us with MOEVE PIC Tracking developed for the simulation of the interaction of bunches with an electron cloud. Further, we would like to thank S. Full (Cornell University) for support especially at the beginning of the project and A. Markovik, G. Hofstaetter and M. Billing (both Cornell University) for fruitful discussions.

[1] G. Hoffstaetter and M. Liepe, Nucl. Instrum. Methods Phys. Res., Sect. A 557, 205 (2006).

[2] F. Zimmermann, T. Raubenheimer, and G. Stupakov, SLAC Report No. SLAC-PUB-95-6792 (1995).

[3] L. Wang, Y. Cai, T. O. Raubenheimer, and H. Fukuma, Phys. Rev. ST Accel. Beams 14, 084401 (2011).

[4] G. Pöplau, MOEVE 2.0: Multigrid Poisson Solver for NonEquidistant Tensor Product Meshes (Universität Rostock, Rostock, Germany, 2006).

[5] A. Markovik, G. Pöplau, and U. van Rienen, in Proceedings of the International Particle Accelerator Conference, Kyoto, Japan (ICR, Kyoto, 2010), p. 1928.

[6] A. Markovik, Ph.D. thesis, Rostock University, 2013.

[7] G. Pöplau, U. van Rienen, S. van der Geer, and M. de Loos, IEEE Trans. Magn. 40, 714 (2004).

[8] G. Pöplau, A. Meseck, and U. van Rienen, in Proceedings of the 3rd International Particle Accelerator Conference,
New Orleans, LA, 2012 (IEEE, Piscataway, NJ, 2012), p. 283.

[9] A. Knobloch et al., in Proceedings of the 3rd International Particle Accelerator Conference, New Orleans, LA, 2012 (IEEE, Piscataway, NJ, 2012), p. 601.

[10] A. Chao, Stanford Linear Accelerator Center Report No. SLAC-PUB-9574, 2002.

[11] Y. Baconnier, CERN Report No. CERN-PS-84-24-PSRREV-2, 1985.

[12] A. Markovik, G. Pöplau, U. van Rienen, D. Sauerland, W. Hillert, and A. Meseck, in Proceedings of the 5th International Particle Accelerator Conference, Dresden, Germany, 2014 (JACoW, 2014), p. 1668.

[13] A. Poncet, in Frontiers of Particle Beams: Factories with e+ and e-Rings, Lecture Notes in Physics, Vol. 425, edited by M. Dienes, M. Month, B. Strasser, and S. Turner (Springer, Berlin, 1994), p. 202.

[14] A. Poncet, in Frontiers of Particle Beams: Intensity Limitations, Lecture Notes in Physics, Vol. 400, edited by M. Dienes, M. Month, and S. Turner (Springer, Berlin, 1992), p. 488.

[15] R. Hockney and J. Eastwood, Computer Simulation Using Particles (Institute of Physics Publishing, Bristol, 1992).

[16] K. Flöttmann, ASTRA, DESY, Hamburg, www.desy.de/ $\sim$ mpyflo (2000).

[17] L. Wang and M. Pivi, in Proceedings of the 3rd International Particle Accelerator Conference, New Orleans, LA, 2012 (IEEE, Piscataway, NJ, 2012), p. 3150.

[18] V. Dürr (private communication).

[19] A. Meseck, G. Pöplau, and U. van Rienen, in Proceedings of the 11th International Computational Accelerator Physics Conference, Rostock, Germany (JACoW, 2012), p. 146. 